\title{
Augmented Standardized Patients Now Virtually a Reality
}

\author{
Frederic D. McKenzie, Hector M. Garcia, Reynel J. Castelino \\ Virginia Modeling Analysis and Simulation Center (VMASC) \\ Old Dominion University \\ fmckenzi@ece.odu.edu,rcast008@,odu.edu,hgarcia@,odu.edu
}

\author{
Thomas W. Hubbard, John A. Ullian, Gayle A. Gliva \\ Theresa A. Thomas Professional Skills Teaching \& Assessment Center \\ Eastern Virginia Medical School \\ HubbarTW@evms.edu, UllianJA@evms.edu,GlivaGA@evms.edu
}

\begin{abstract}
Standardized patients (SPS), individuals who realistically portray patients, are widely used in medical education to teach and assess communication skills, eliciting a history, performing a physical exam, and other important clinical skills. One limitation is that each SP can only portray a limited set of physical symptoms. Finding SPS with the abnormalities students need to encounter is typically not feasible. This project augments the SP by permitting the learner to hear abnormal heart and lung sounds in a normal SP.
\end{abstract}

\section{Introduction}

To become clinically competent physicians, medical students must develop knowledge and skills in many areas of both the art and science of medicine. Three areas are emphasized in medical students' early clinical training: doctor-patient communication, eliciting the history, and performing the physical exam. Standardized patients (SPs), individuals trained to realistically portray patients, are commonly used to teach and assess medical students in those three areas. Working with them provides students the opportunity to learn doctor-patient communication, the history, the physical exam, and other clinical skills in a safe setting. SPs also provide a way to reliably test students' clinical skills in a realistic setting, interacting with a person. The range of clinical problems an SP can portray, however, is limited. They are typically healthy individuals with few or no abnormal physical findings. While some can be trained to simulate physical abnormalities (e.g., breathing through one lung, voluntarily increasing blood pressure, etc.), there are many abnormalities they cannot simulate.

One way to supplement what students learn from SPs is for the students to separately learn from and practice on simulators. A variety of mechanical or computer-based simulators are now used in medical education, including software for testing clinical reasoning and diagnostic skills, computer simulations of physiological processes, and physical models for practicing selected procedural skills. A key limitation is that their users are isolated from interacting with a live person (the patient or SP) while using the simulators. Augmenting SPs with the ability to simulate abnormal physical findings would expand the opportunities for students to learn more clinical skills in a realistic setting with a real person (SP) while practicing their doctor-patient communication skills.

The current phase of this research involves simulating abnormal heart or lung sounds in an SP, thus expanding the breadth of sounds that can be heard in an SP. A learner will listen to an SP's heart and lungs through a modified stethoscope and hear pre-recorded sounds rather than the SP's. With a real or standardized patient, the learner is limited to hearing only the sounds of that single person. Learning a variety of sounds has traditionally required examining many patients over time, often without direct supervision and feedback. Commercially available recordings of heart and lung sounds exist, but using them ignores the process of listening for the sounds (e.g., correct placement of the stethoscope) and excludes simultaneous interactions with the patient. Augmenting SPs with the capability of portraying patients with an increased range of abnormalities will make the use of SPs an even more valuable teaching tool.

\section{Methods}

Augmented reality integrates text, graphics, sounds, etc. with the natural environment in order to enhance or inform the experience. We have begun by constructing a functional prototype that augments the reality of the SP with virtual sounds for his/her heart and lung. The intent is to impart abnormal pathology that could not be faked or reproduced by the SP alone. This would greatly enhance the repertoire of lessons that could be hands-on trained by the SP and not for their first time on actual patients. 


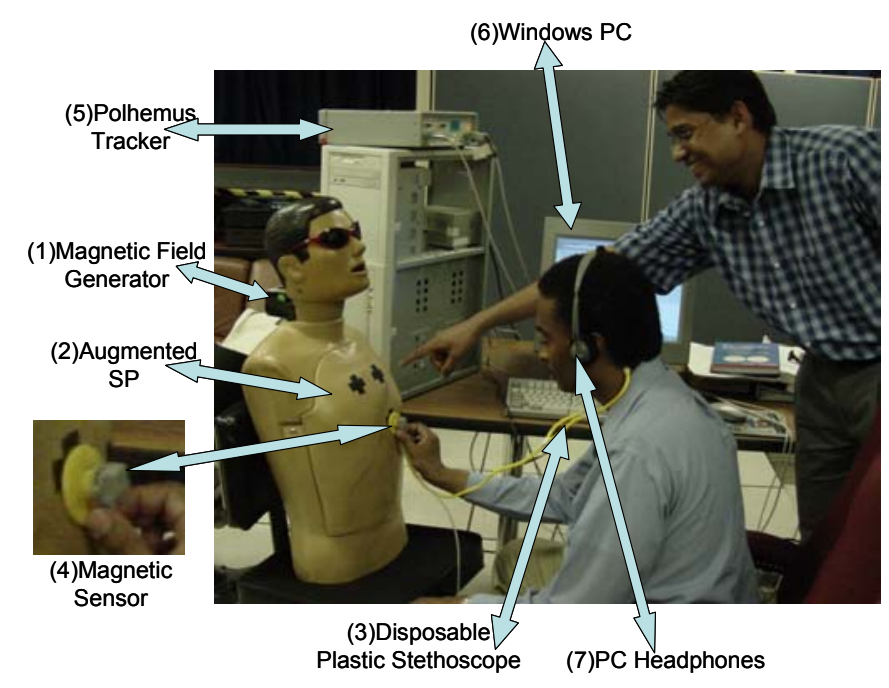

Figure 1. Prototype System

Our simulation involved the use of a mannequin, labeled in Figure 1 as the (2) Augmented SP, fitted with an electromagnetic field generator (1) and a movable sensor connected to the stethoscope head (4). We used the 3SPACE FASTRAK from POLHEMUS as the tracker and the trackd API from VRCO as the PC interface to obtain real time 6 DoF updates of position $(\mathrm{X}, \mathrm{Y}$, and $\mathrm{Z}$ Cartesian coordinates) and orientation (azimuth, elevation, and roll) [1]. Essentially, the system operates as follows. The medical student would use the disposable stethoscope (3) to place against the Augmented SP and perform auscultation. As the student places the stethoscope head, its position is tracked via the Polhemus FASTRAK (5) using the movement of the attached sensor. When the system software running on the PC (6) detects that the sensor / stethoscope head is placed within an appropriate location, the software triggers the corresponding sound file which plays into the headphones (7) that the student is wearing. Everything occurs in real time and the sounds trigger according to the "hot zones" marked by black crosses on the mannequin.

\section{Conclusions}

A useable augmented standardized patient system is virtually a reality. We have demonstrated its usefulness by virtue of a proof of concept prototype. A completely virtual SP has been tried before by Hubal et al [2]. They utilize natural language processing, and virtual patients that exhibit emotion in a realistic context to provide completely automatic yet unscripted training sessions. The motivation of the researchers was in replacing the SP because of concerns such as the cost in paying and training actors as well as quality control and reproducibility of a session. These concerns are genuine; however, human-computer interaction brings a different set of psychological concerns than does the humanhuman interaction of a doctor-patient examination. A significant level of immersion is needed to overcome the human-computer interaction aspects so that there is appreciable transfer of training with regards to patient interaction and diagnosis. This level of immersion and interactivity has not been reached and may not be achievable in a totally virtual form with today's technology. Although Hubal et al provide a useful tool for outlining the steps for patient interaction and diagnosis, our augmented SP work seeks to provide a completely realistic experience by greatly enhancing a currently accepted medium of instruction.

Our project was successful in its attempt to allow listening to pre-recorded heart and lung sounds when the head of the modified stethoscope was placed at any of four locations on a mannequin torso. There is nothing special in the use of the mannequin. A real human (SP) could have been augmented with our system just as easily if she had the inclination to stay still for long periods of time during our testing. The initial proof-of-concept evaluation of the system was performed by an EVMS doctor experienced in SPs and the training of auscultation. Even with the limited functionality of this first prototype, it was evident that the concept works and with a bit of tracking refinement and expanded fields of interest with abnormalities, the system could become a useful and integral part of auscultation education.

\section{Acknowledgements}

This study was a collaborative project between the Virginia Modeling, Analysis and Simulation Center (VMASC) at Old Dominion University and the Eastern Virginia Medical School. Funding for this study was provided in part by the Naval Health Research Center through NAVAIR Orlando TSD under contract N61339-03-C-0157, entitled "The National Center for Collaboration in Medical Modeling and Simulation". The ideas and opinions presented in this paper represent the views of the authors and do not necessarily represent the views of the Department of Defense.

\section{References}

[1] www.polhemus.com

[2] Hubal, R.C., Kizakevich, P.N., Merino, K.D., \& West, S.L. The Virtual Standardized Patient: Simulated PatientPractitioner Dialogue for Patient Interview Training. Envisioning Healing: Interactive Technology and the Patient-Practitioner Dialogue. J. Westwood, H. Hoffman, G. Mogel, R. Robb, \& D. Stredney (Eds.), IOS Press: Amsterdam, 2000. 